\title{
Immunostimulatory oncolytic virotherapy for multiple myeloma targeting 4-1BB and/or CD40
}

\author{
Jessica Wenthe $\mathbb{B D}^{1} \cdot$ Sedigheh Naseri ${ }^{1} \cdot$ Ann-Charlotte Hellström ${ }^{1} \cdot$ Helena Jernberg Wiklund ${ }^{1} \cdot$ Emma Eriksson $^{1}$ \\ Angelica Loskog $\mathbb{B}^{1,2}$
}

Received: 2 March 2020 / Revised: 2 April 2020 / Accepted: 15 April 2020 / Published online: 1 May 2020

(c) The Author(s) 2020. This article is published with open access

\begin{abstract}
Multiple myeloma (MM) is a plasma cell malignancy that is characterized by immune dysregulation. MM is commonly treated with immunomodulating agents, but still remains incurable. Herein, we proposed and evaluated immunostimulatory Lokon oncolytic adenoviruses (LOAd) for MM treatment. LOAd viruses are serotype 5/35 chimera, which enables infection of hematopoietic cells. Oncolysis is restricted to cells with a dysregulated retinoblastoma protein pathway, which is frequently observed in MM. Further, LOAd viruses are armed with human immunostimulatory transgenes: trimerized membrane-bound CD40L (LOAd700, LOAd703) and 4-1BBL (LOAd703). LOAd viruses were assessed in a panel of MM cell lines (ANBL-6, L363, LP-1, OPM-2, RPMI-8226, and U266-84). All cells were sensitive to infection, leading to viral replication and cell killing as analyzed by quantitative PCR and viability assay. Transgene expression was verified post infection with flow cytometry. Cell phenotypes were further altered with a downregulation of markers connected to MM progression (ICAM-1, CD70, CXCL10, CCL2, and sIL-2R $\alpha$ ) and an upregulation of the death receptor Fas. In a co-culture of immune and MM cells, LOAd viruses promoted activation of cytotoxic T cells as seen by higher CD69, CD107a, and IFN $\gamma$ expression. This was most prominent with LOAd703. In conclusion, LOAd viruses are of interest for MM therapy.
\end{abstract}

\section{Introduction}

Multiple myeloma (MM) is a plasma cell malignancy and the second most common hematological cancer type with an incidence rate of 5 cases per 100,000 people in the Western world. Even though current treatment options have improved the overall survival to a median of 6 years, MM still remains incurable [1,2]. Patients with high-risk MM with presence of extramedullary disease (infiltrates in soft tissue or visceral organs) have an especially bad prognosis with no effective treatment options available [3, 4]. Thus, the development of novel therapies is of particular importance for this patient

Supplementary information The online version of this article (https:// doi.org/10.1038/s41417-020-0176-9) contains supplementary material, which is available to authorized users.

Jessica Wenthe

jessica.wenthe@igp.uu.se

1 Department of Immunology, Genetics and Pathology, Science for Life Laboratory, Uppsala University, Uppsala, Sweden

2 Lokon Pharma AB, Uppsala, Sweden group. Immunotherapies, such as immune checkpoint inhibition and chimeric antigen receptor $\mathrm{T}$ cell therapy, have shown great success in a variety of cancers, but these approaches seem to be more challenging in MM [5]. Nevertheless, considering that an important hallmark of MM is immune dysregulation, which facilitates the escape from immune surveillance due to impaired dendritic cell (DC) function and Th1 responses, it is especially intriguing to advance immunotherapeutic approaches for this indication [6]. Specifically, oncolytic virotherapy represents a suitable option as oncolytic viruses are able to enhance the immunogenicity of tumors and induce antitumor immune responses [7]. In addition, MM cells often display overexpression of viral entry receptors (e.g., CD46) and alterations in signaling pathways, which enables viral infection and replication. Previous preclinical and clinical studies in MM have mostly utilized measles virus, reovirus, or vesicular stomatitis virus $[8,9]$. Herein, we are investigating oncolytic adenoviruses within the Lokon oncolytic adenovirus (LOAd) platform in human MM models. Commonly used serotype 5 adenoviruses cannot efficiently infect hematopoietic cells due to absence of the coxsackievirus and adenovirus receptor in these cells [10]. LOAd viruses are serotype chimera, meaning 
that the adenoviral fiber and knob are switched to serotype 35 (Ad5/35) [11]. This retargets the virus to infect CD46+ cells, thereby enabling infection of most cell types, including MM cells. Viral replication is controlled due to a deletion in the viral E1A site (E1A $\Delta 24)$, thereby restricting lysis to cells with a dysregulated retinoblastoma pathway. Intriguingly, many MM patients display even complete deletions of the retinoblastoma gene, which should drive potent LOAd replication [12-14]. In addition to their oncolytic function, LOAd viruses are further modified to encode for immunostimulatory transgenes in order to enhance antitumor immune responses. Both LOAd700 [11] and LOAd703 [15] encode a designed human trimerized membrane-bound CD40L, but LOAd703 additionally encodes for human wildtype full length 4-1BBL. CD40 stimulating therapy may be of special interest for MM since these cells origin from germinal center B cells and thus have similar features as professional antigen-presenting cells [16]. For instance, stimulation of B cell lymphomas with adenoviruses encoding CD40L has been shown to increase their antigen-presentation capacity and ability to activate antigen-specific cytotoxic $\mathrm{T}$ cells $[17,18]$. Interestingly, also $\mathrm{MM}$ cells have shown to react to CD40L gene therapy in a similar manner. For example, Dotti et al. demonstrated that MM cells infected with an adenovirus carrying CD40L could activate bystander DCs, which in turn could induce $\mathrm{T}$ cell responses [19]. Moreover, an oncolytic adenovirus armed with CD40L has been shown to cause enhanced MM cell killing and upregulation of the death receptor Fas [20]. Hence, the aim of this study was to evaluate LOAd viruses armed with CD40L for the treatment of MM by determining the oncolytic and immunostimulatory function of LOAd700 and LOAd703 in preclinical MM models.

\section{Materials and methods}

\section{Cell culture}

The human MM cells lines (ANBL-6, L363, LP-1, OPM-2, RPMI-8226, and U266-84) [21] were provided by Prof Helena Jernberg Wiklund at Uppsala University. All cell lines were maintained in RPMI-1640 supplemented with fetal bovine serum $(10 \%)$, penicillin $(100 \mathrm{U} / \mathrm{ml})$ and streptomycin $(100 \mu \mathrm{g} / \mathrm{ml})$ (all supplements purchased from Thermo Fisher Scientific, Waltham, MA, USA). ANBL-6 medium was further supplemented with $2 \mathrm{ng} / \mathrm{ml}$ IL-6 (BioLegend, San Diego, CA, USA). The cell lines were STR typed and tested negative for mycoplasma contamination with MycoAlert ${ }^{\mathrm{TM}}$ Mycoplasma Detection Kit (Lonza, Basel, Switzerland) prior experiments. In general, cells were thawed and cultured for two to three weeks ( 4-6 passages) before initiation of experiments.

\section{Virus construction and infection}

The construction and expansion of LOAd viruses have been previously described [11]. The viral infectious titers were quantified with a fluorescence-forming unit (FFU) assay [22]. LOAd700 and LOAd703 viruses are both armed with human trimerized membrane-bound CD40L (TMZCD40L), whereas LOAd703 is additionally armed with human 4-1BBL. Transgenes are expressed under the control of a CMV promoter. $\operatorname{LOAd}(-)$, a virus without transgenes, and a replication-deficient adenovirus 5/35 (Ad5/35 Mock; E1/E3 deleted) were used as controls. A schematic figure of all virus constructs is shown in Supplemental Fig. S1. LOAd viruses were provided by Lokon Pharma $A B$, Uppsala, Sweden. For virus infection, cells were washed in serum-free medium and pelleted before the respective virus was added (10-200 multiplicity of infection $(\mathrm{MOI})=$ FFU/ cell). Cell suspensions were incubated at $37^{\circ} \mathrm{C}, 5 \% \mathrm{CO}_{2}$ for $2 \mathrm{~h}$ followed by the addition of complete growth medium.

\section{Cell viability assay}

Cells were infected with the respective virus or left uninfected. Two hours after infection, $1 \times 10^{4}$ cells/well were plated in 96-well plates in quadruplicates. Cells were analyzed for their viability $72 \mathrm{~h}$ post infection with CellTiter 96 AQueous One Solution MTS reagent (Promega, Fitchburg, WI, USA) according to manufacturer's instructions.

\section{DNA isolation and quantitative PCR}

Viral DNA was isolated at 2, 24, 48, and $96 \mathrm{~h}$ after virus infection using High Pure Viral Nucleic Acid kit (Roche, Basel, Switzerland). Viral replication was assessed using quantitative (q)PCR with primers detecting the adenoviral E4 orf1 transcript (Fw- 5'CATCAGGTTGATTCACATCGG; Rw- 5'GAAGCGCTGTATGTTGTTCTG) [23]. QPCR was performed using iQ SYBR $^{\circledR}$ Green PCR Supermix kit (Bio-Rad, Hercules, CA, USA) and 1:100 diluted viral DNA. The PCR products were continuously measured by the Bio-Rad CFX96 Real-Time detection system for 40 cycles.

\section{In vivo experiments}

Animal experiments were approved by local animal ethics committee (Dnr: 5.8.18-13471/2017) and performed at Uppsala University. Cells from the human MM cell line RPMI8226 mixed 1:1 with Matrigel (Corning, NY, USA) were injected subcutaneously in immunodeficient BALB/c nude mice $\left(5 \times 10^{6}\right.$ cells/mouse, five mice per group). Palpable tumors (8-10 days post tumor injection) were 
injected 6x (twice per week) with LOAd(-) or LOAd703 $\left(1 \times 10^{9} \mathrm{FFU}\right)$ or phosphate buffered saline (PBS)/saline as negative control. Mice were treated either intratumorally or intravenously depending on the experimental set-up. Tumor growth was monitored by measuring the tumor area (width by height) and mice with tumors over $100 \mathrm{~mm}^{2}$ were sacrificed.

\section{Flow cytometry analysis of immune markers}

Cells were infected with the respective virus (100 FFU/cell) or left uninfected. Forty-eight hours after infection, cells were harvested and washed in PBS supplemented with $3 \mathrm{mM}$ EDTA (Thermo Fisher, Waltham, USA) and 0.5\% bovine serum albumin (Sigma-Aldrich, Saint Louis, MO, USA). Cells were stained with fluorescent-labeled antibodies targeting CD46 (PE; clone TRA-2-10), CD40L (BV421; clone 24-31), 4-1BBL (PE; clone 5F4), CD40 (APC; clone HB14), 4-1BB (BV421; clone 4B4-1), HLA-ABC (FITC; clone G46-2.6), HLA-DR (APC; clone L243), CD86 (BV421; clone IT2.2), CD70 (PE; clone 113-16), ICAM-1 (FITC; clone HCD54), and Fas (FITC; clone DX2). All antibodies were purchased from BioLegend. After staining, cells were fixed in PBS containing 1\% formaldehyde and $3 \mathrm{mM}$ EDTA and analyzed with BD FACS Canto 2 (BD Biosciences, San Jose, CA, USA). The data were evaluated using FlowJo software (FlowJo LLC, Ashland, OR, USA).

\section{Detection of soluble immune markers}

For detection and quantification of soluble immune markers, supernatants from infected $(100 \mathrm{ffu} / \mathrm{cell})$ and uninfected cells were collected at $48 \mathrm{~h}$ of post infection and analyzed with a MSD multiplex U-PLEX assay (Meso Scale Discovery, Rockville, MD, USA) according to the manufacturer's protocol.

\section{Stimulation of peripheral blood mononuclear cells (PBMCs) in multiple myeloma co-cultures}

PBMCs were isolated by density centrifugation with FicollPaque (GE Healthcare, Chicago, IL, USA) from healthy donor buffy coats acquired from the blood bank at Uppsala University hospital. PBMCs were cultured alone or cocultured either with L363 or U266-84 cells in a ratio of 2:1 at a concentration of $1 \times 10^{6}$ cells $/ \mathrm{mL}$ in 6 -well plates $(3 \times$ $10^{6}$ cells in total). After $24 \mathrm{~h}$, cells were infected with the respective virus by direct addition of virus into the respective well (100 FFU/cell). PBMCs cultured alone and one co-culture well were left uninfected. Forty-eight hours of post infection, cells were harvested and cell culture supernatants were taken for IFN- $\gamma$ detection (Human IFN- $\gamma$ ELISA development kit, Mabtech AB, Nacka Strand,
Sweden). Cells were stained for flow cytometry analysis as described above with the following antibodies purchased from BD Biosciences: CD45 (FITC; clone 2D1), CD16 (PE; clone B73.1), CD56 (PE; clone My31), CD3 (PerCP; clone SK7), CD4 (PE-Cy7, APC-H7; clone SK3), CD8 (APC; SK1), CCR7 (FITC; clone 150503), CD45RA (APCH7; clone HI100), PD-1 (PE; clone EH12.1), CD69 (FITC; clone L78), CD25 (PE, clone 2A3), CD127 (PE-Cy7; HIL7R-M21). CD3 (PerCP; clone UCHT1), CD8a (FITC; clone RPA-T8), and CD107a (PE; clone H4A3) were purchased from BioLegend.

\section{Statistical analysis}

GraphPad Prism 8 (GraphPad Software, San Diego, CA, USA) was used for the statistical analysis. For animal experiments, statistically differences in tumor size were analyzed with Kruskal-Wallis test followed by Dunn's multiple comparison test. Differences in survival were calculated with Log-rank test. For PBMC co-culture experiments, Friedman test followed by Dunn's multiple comparisons test was used.

\section{Results}

\section{LOAd platform viruses kill and replicate in human multiple myeloma cell lines}

A panel of six human MM cell lines (ANBL-6, L363, LP-1, OPM-2, RPMI-8226, and U266-84) was selected for the evaluation of LOAd platform viruses for the treatment of MM. LOAd viruses infect cells via the complement regulator CD46, which is frequently overexpressed in many cancer types including MM [9, 24]. All chosen MM cell lines were confirmed to express CD46 with a highest expression level of $96 \%$ in RPMI-8226 cells (range in all cell lines: 42-96\% CD46+) (Fig. 1a). To determine the oncolytic capacity of LOAd viruses, MM cell lines were infected with LOAd viruses or replication-deficient Ad5/35 Mock virus (E1/E3 deleted) at different virus to cell ratios (10, 20, 50, and $200 \mathrm{MOI})$. All cell lines were efficiently killed by LOAd viruses $72 \mathrm{~h}$ of post infection (Fig. 1b). ANBL-6 was the least sensitive cell line, whereas all other cell lines were also sensitive to Mock virus infection, especially at a higher MOI. OPM-2 appeared especially sensitive as Mock virus killed the cells to a similar extent as LOAd viruses. However, the sensitivity did not seem to correlate with CD46 expression as both ANBL-6 and OPM2 expressed CD46 to a higher degree (83 and 89\%, respectively). To verify that LOAd-infected cells were indeed killed by oncolysis, viral replication was assessed by quantitative PCR. LOAd viruses were shown to replicate in 
A.

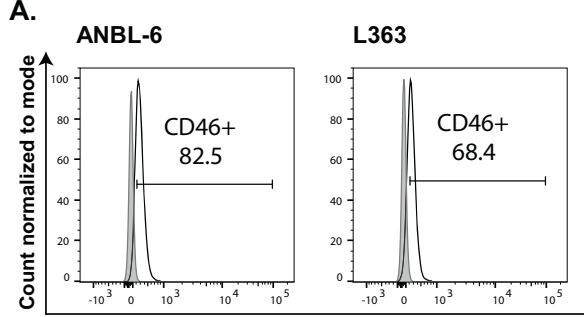

LP-1

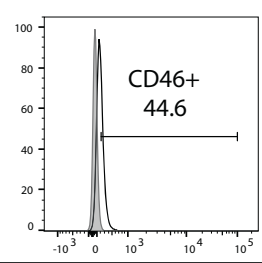

OPM-2

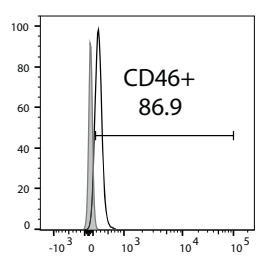

RPMI-8226

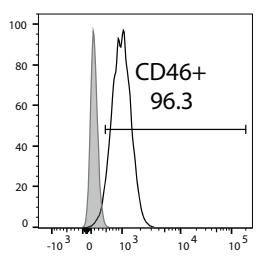

U266-84

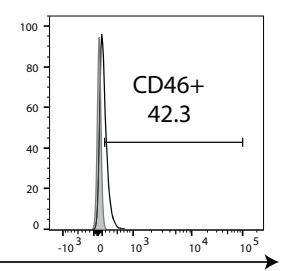

B.
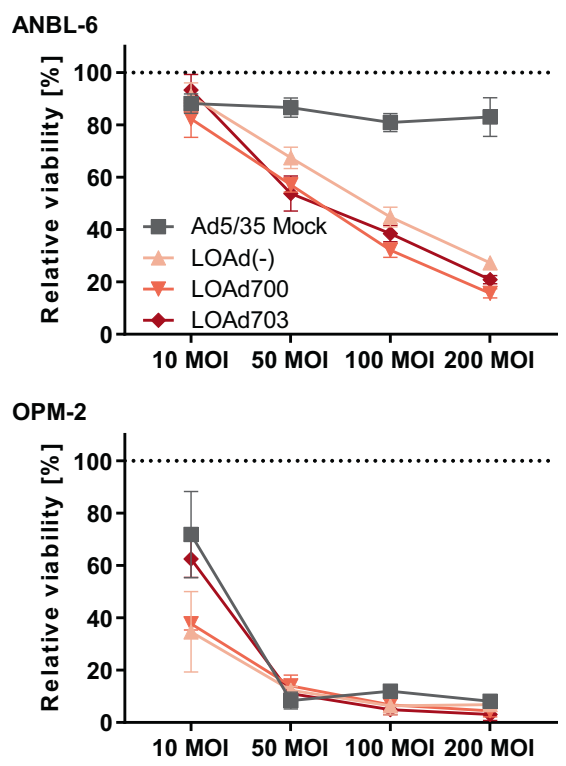

L363

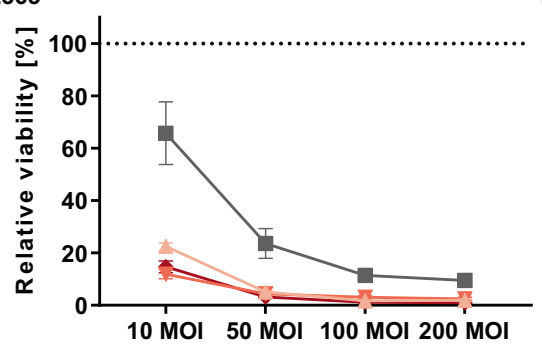

RPMI-8226

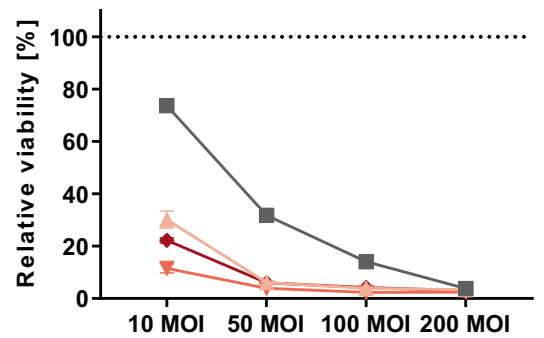

LP-1

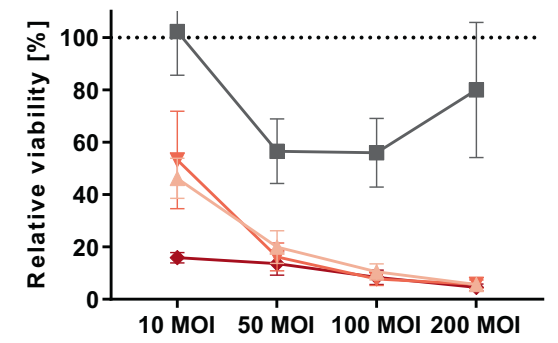

U266-84

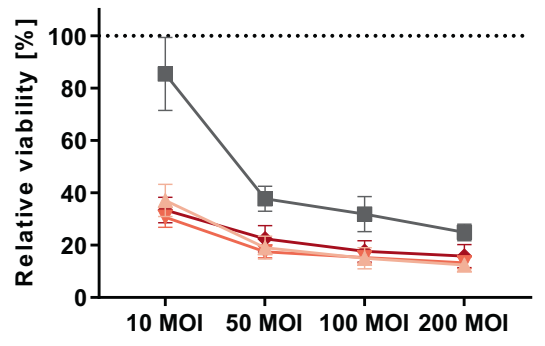

Fig. 1 Multiple myeloma cell lines express CD46 and are killed by LOAd viruses. Human multiple myeloma cell lines ANBL-6, L363, LP-1, OPM-2, RPMI-8226, and U266-84 were analyzed for their expression of CD46 by flow cytometry. Gray filled histograms represent matched isotype controls and black lines represent CD46 staining (a). All abovementioned cells lines were infected with
LOAd viruses or replication-deficient Ad5/35 Mock virus at different virus to cell ratios $(10,20,50$, and $200 \mathrm{MOI})$. Cell viability was analyzed with MTS viability assay at $72 \mathrm{~h}$ post infection and viability is shown as percentage viability of uninfected control cells. $n=2$; technical replicates $=4$; graphs show mean \pm SEM. all cell lines as shown by a 100-1000-fold increase of viral DNA over time compared to $2 \mathrm{~h}$ of post infection (Fig. 2). In comparison to all other cell lines, viral replication was lowest in U266-84 cells, leading only to a maximum 10fold increase of viral DNA. Of note, an increase of viral DNA was also detected in Mock-infected cells in 5 out of 6 cell lines, but this increase was much lower (circa 3-30fold) compared to LOAd-infected cells and may be due to presence of wild type virus (replication competent adenovirus (RCA)) that is often present in adenovirus batches but at low frequencies [25].

\section{LOAd703 controls tumor growth in a multiple myeloma xenograft model}

To investigate the oncolytic function of LOAd viruses in vivo, a xenograft mouse model with subcutaneously injected RPMI-8226 cells was used. Due to fact that adenoviruses are non-enveloped viruses that can be readily neutralized by antibodies, LOAd viruses were so far only evaluated in vivo with intratumoral injections in solid tumors $[11,15]$. Nevertheless since MM is a systemic disease, we also wanted to explore intravenous treatments. Note, the effect of the human transgenes cannot be evaluated in a xenograft model since nude mice lack an adaptive immunity and human CD40L cannot activate murine CD40. However, there is a minimal cross reactivity of human 41BBL with murine 4-1BB [26]. Hence, to confirm that the oncolytic function of armed LOAd viruses are not impaired in vivo, only LOAd703, which encodes for both CD40L and 41BBL, was used and compared to unarmed LOAd( - ). Mice with established tumors (day 8-10 post tumor injection) were treated in total six times (two treatments per week) with PBS/saline, $\operatorname{LOAd}(-)$ or LOAd703 either intratumorally (Fig. 3a, b) or intravenously (Fig. 3c, d). Intratumoral injections of both $\operatorname{LOAd}(-)$ and LOAd703 were able to control tumor growth (Fig. 3a) compared with PBS control (PBS vs. $\operatorname{LOAd}(-) p=0.0115$, PBS vs. LOAd703 $p=0.0098)$. Moreover, LOAd703-treated mice demonstrated a prolonged survival as compared to the PBS 


\section{A. ANBL-6}

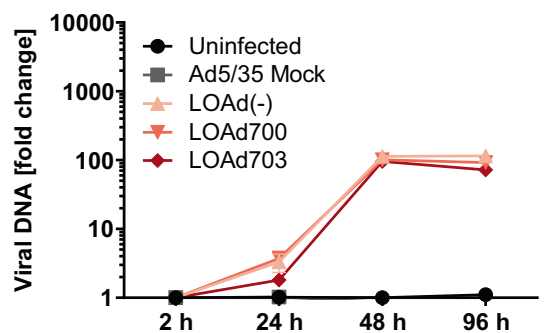

\section{OPM-2}

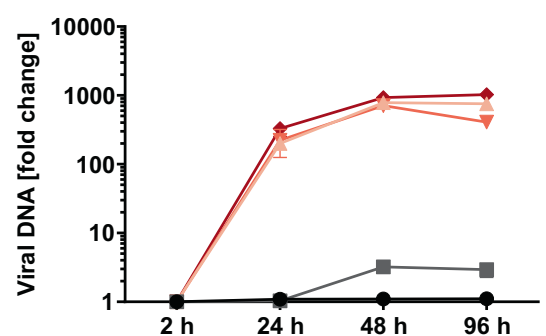

B. L363

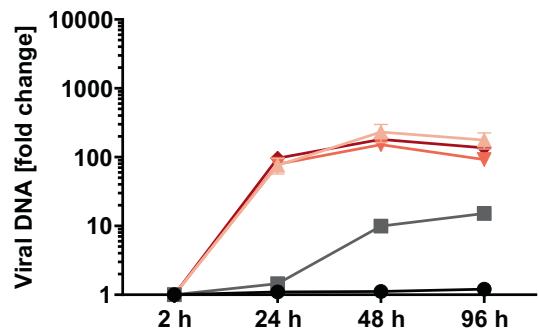

E. RPMI-8226

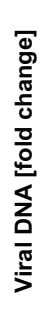

C. LP-1

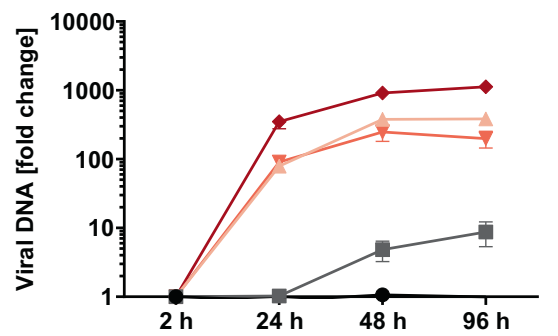

F. U266-84

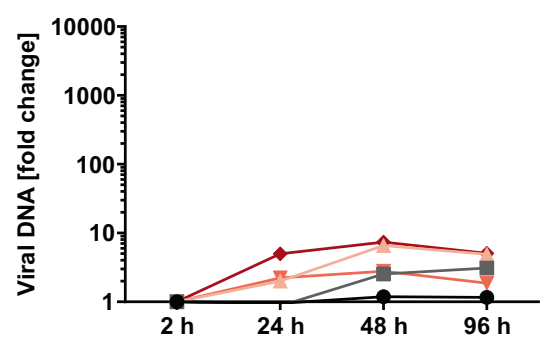

Fig. 2 LOAd viruses replicate in multiple myeloma cells. Human multiple myeloma cell lines (ANBL-6 (a), L363 (b), LP-1 (c), OPM-2 (d), RPMI-8226 (e), and U266-84 (f)) were infected with 50 MOI of LOAd viruses or replication-deficient Ad5/35 Mock virus. Viral DNA was isolated at $2,24,48$, and $96 \mathrm{~h}$ post infection and quantified with primers detecting adenoviral DNA. Graphs display the fold change of viral DNA compared to baseline ( $2 \mathrm{~h}$ post infection). $n=2$; technical replicates $=3$; graphs show mean \pm SEM.
A.

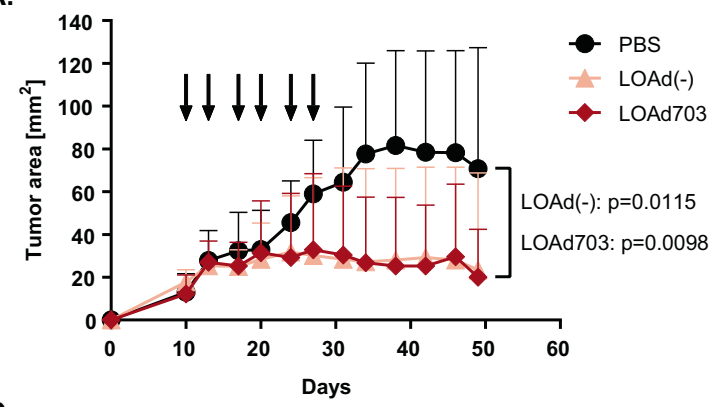

c.

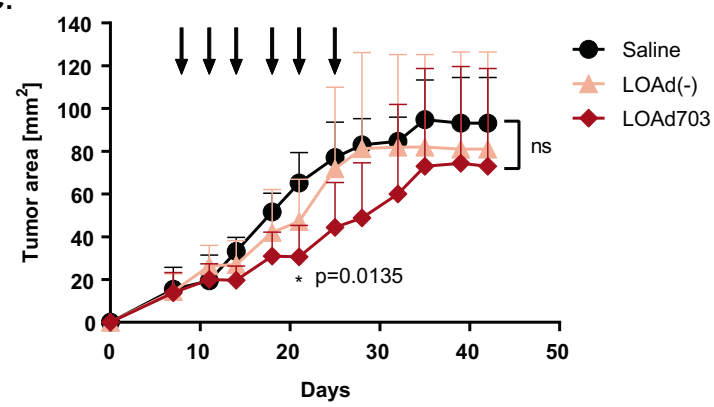

B.

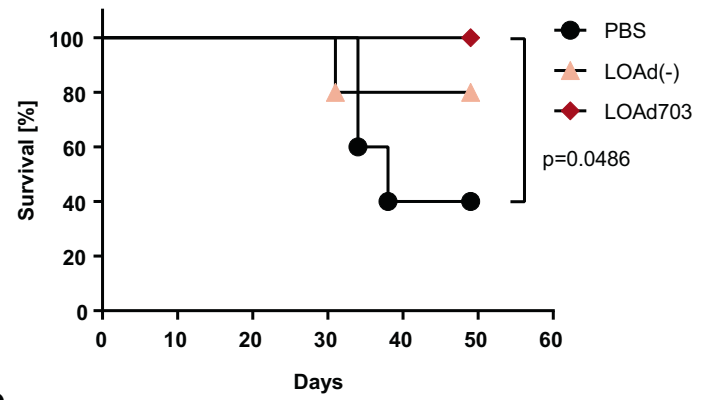

D.

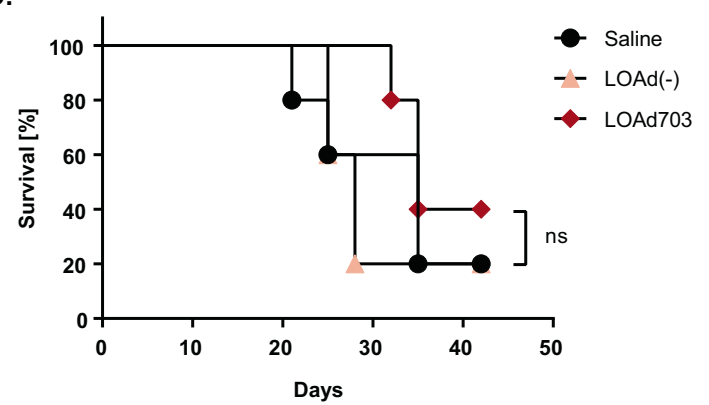

intratumorally $(\mathbf{a}, \mathbf{b})$ or intravenously $(\mathbf{c}, \mathbf{d})$. Mean tumor size + SD is displayed in graphs a and c. Survival curves are shown in $\mathbf{b}$ and $\mathbf{d}$. Statistically differences in tumor size were analyzed with Kruskal-Wallis test followed by Dunn's multiple comparisons test. Differences in survival were calculated with Log-rank test.

points (Fig. 3c). Likewise, there was no survival benefit in LOAd703-treated mice, but 2/5 mice of this group were alive at the end of experiment (day 42) compared with $1 / 5$ mice in the other two groups (Fig. 3d) 


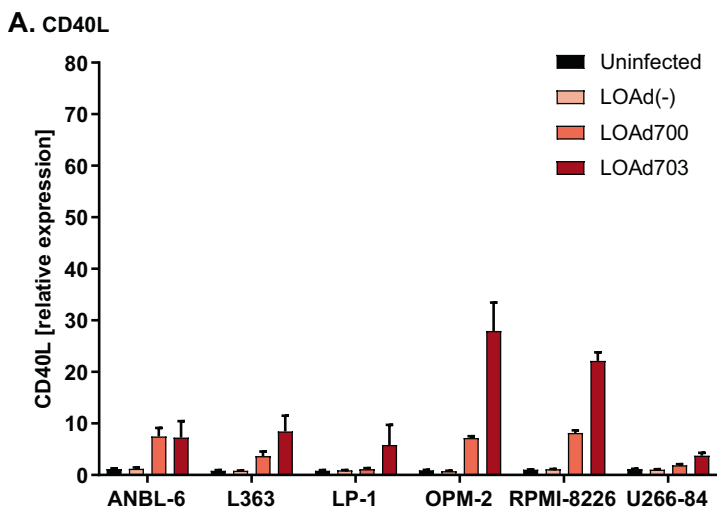

Fig. 4 Transgene expression in LOAd-infected multiple myeloma cells. Cells were infected with $100 \mathrm{MOI}$ of $\operatorname{LOAd}(-), \operatorname{LOAd700}$, and LOAd703 or left uninfected and cultured for $48 \mathrm{~h}$ before cells were harvested for transgene expression analysis by flow cytometry. Bar

\section{LOAd infection induces transgene expression in all cell lines}

LOAd platform viruses are further modified to encode for human immunostimulatory transgenes, which will be expressed in infected cells independent of virus replication. Both LOAd700 and LOAd703 are modified to encode for a designed trimerized membrane-bound CD40L [11], but LOAd703 additionally encodes 4-1BBL [15]. The control virus $\operatorname{LOAd}(-)$ contains no transgenes. The inherent baseline expression of CD40L and 4-1BBL and the corresponding receptors $\mathrm{CD} 40$ and $4-1 \mathrm{BB}$ in the cell lines is shown in Supplemental Figs. S2-S7. CD40L and was not expressed in any of the uninfected cells, whereas 4-1BBL was expressed to a low extent in all cell lines. CD40 and 41BB could not be detected in any of the cells apart from RPMI-8226 cells, which displayed a minor CD40 expression $(\sim 6 \% \mathrm{CD} 40+)$. Upon LOAd infection (100 MOI), the respective transgenes were expressed in all MM cell lines as analyzed by flow cytometry and compared to matched isotype controls (Fig. 4). LOAd703 induced higher levels of CD40L than LOAd700 (Fig. 4a), but CD40L expression was overall lower than 4-1BBL expression (Fig. 4b).

\section{Immune markers on multiple myeloma lines post LOAd infection}

MM cells are derived from germinal center B cells and can express markers for antigen presentation and $\mathrm{T}$ cell activation. We evaluated if such markers were present and if their expression was altered by LOAd infection. Upregulation of MHC, costimulatory, and adhesion molecules may enable antigen presentation by $\mathrm{MM}$ cells and subsequent $\mathrm{T}$ cell stimulation, which has been seen by others with induced CD40L expression by adenoviruses [19]. All MM cell lines

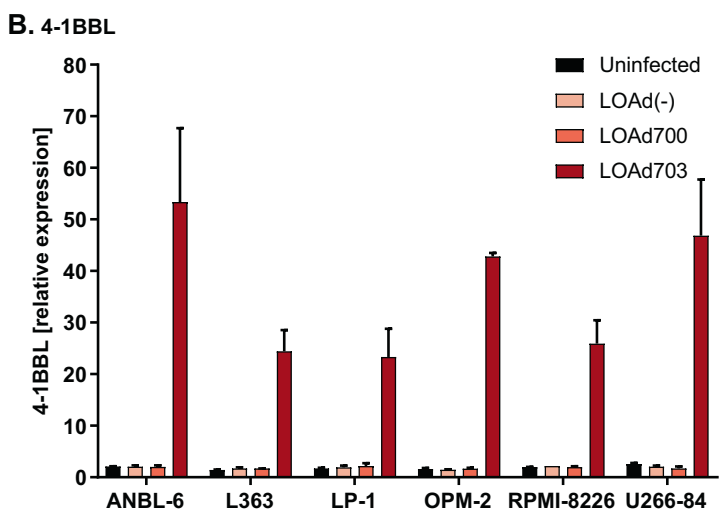

graphs display the geometric mean fluorescent intensity (MFI) of CD40L (a) and 4-1BBL (b) divided by the MFI of matched isotype control antibodies (relative expression). $n=2$; mean \pm SEM.

expressed MHC class I molecules (HLA-ABC) while only three of six (L363, LP-1, and U266-84) displayed MHC class II (HLA-DR) (Fig. 5a, b). Costimulatory and adhesion molecules such as CD86, CD70, and ICAM-1 were present to varying degrees on all lines tested (Fig. $5 \mathrm{c}-\mathrm{e}$ ). The markers were commonly decreased post virus infection, which was more prominent after infection with LOAd700 or LOAd703 that encodes transgenes. Hence, virus replication and expression of transgenes may somehow compete with the cell transcription machinery in these cells due to the oncolysis process, or the transgenes may have a regulatory capacity in MM cells. The same types of markers are upregulated post infection with LOAd viruses in other antigen-presenting cells such as DCs [15]. Interestingly, the apoptosis receptor Fas was upregulated post virus infection in 5 out of 6 cell lines (Fig. 5f).

\section{LOAd viruses promote CCL3 expression in multiple myeloma lines}

The presence of soluble immune markers that are frequently implicated in MM [27-30] was analyzed pre-LOAd and post-LOAd infection of MM cells using MSD multiplex assay. The analytes IFN $\gamma$, IL-1RA, CCL22, CCL17, and TNF $\alpha$ were undetectable in ANBL-6, L363, and OPM-2 and only present at low amount and to a varying degree in the other cell lines (Supplemental Fig. S8). However, most lines expressed CXCL10, IL-8, CCL2, sIL-2R $\alpha$, and CCL3 (Fig. 6). Overall, these soluble markers were decreased post infection that can be due to the rapid onset of the oncolysis process, which ultimately leads to less time to release these markers. However, CCL3 was increased by LOAd infection, except in RPMI-8226 cells (Fig. 6e). Contrarily, RPMI-8226 cells had increased levels of IL-8 post infection of LOAd700 and LOAd703 (Fig. 6b). 
A. HLA-ABC

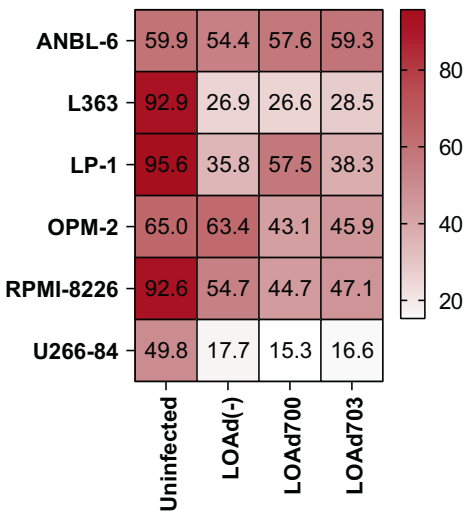

D. CD70

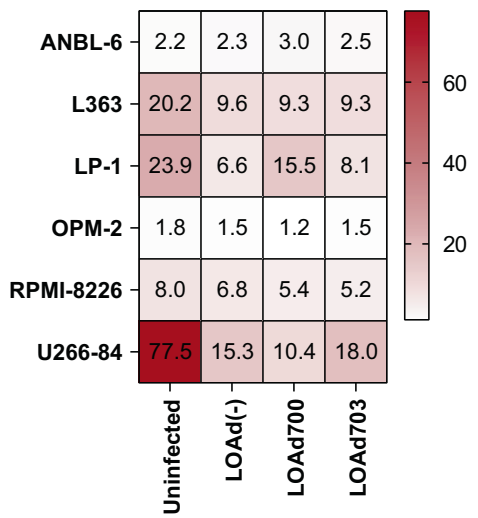

B. HLA-DR

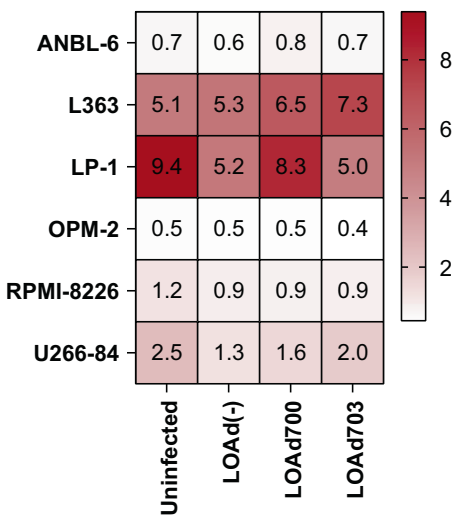

E. ICAM-1

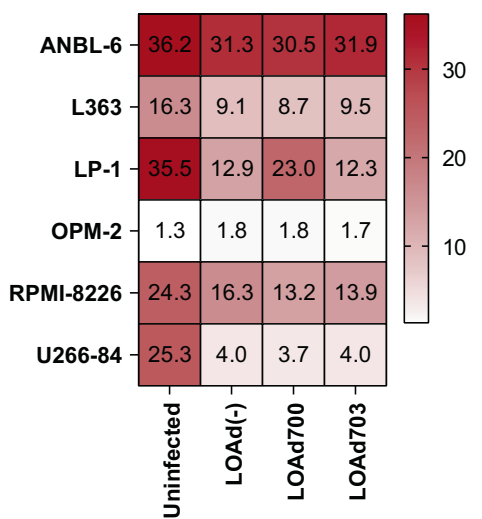

C. $\operatorname{CD} 86$

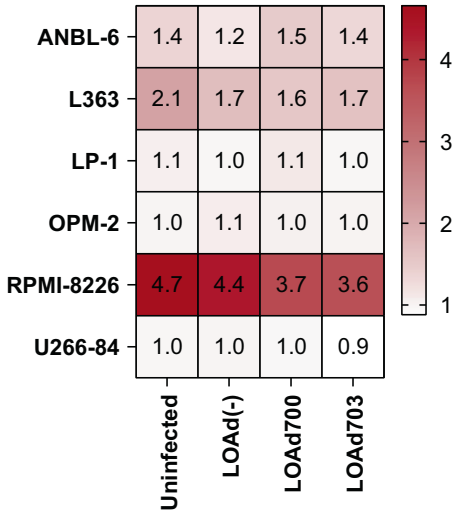

F. Fas

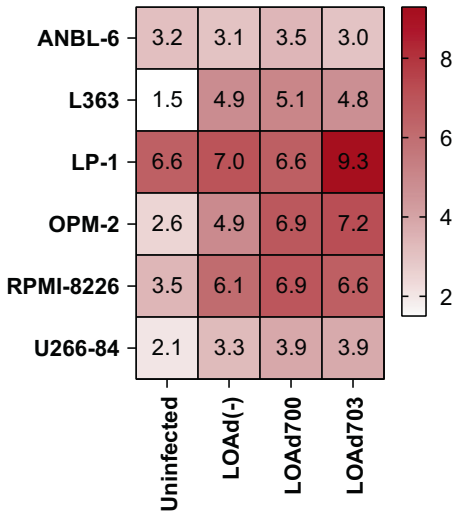

Fig. 5 Expression levels of immune markers on LOAd-infected multiple myeloma cells. Cells were infected with 100 MOI of LOAd (-), LOAd700, and LOAd703 or left uninfected, cultured for $48 \mathrm{~h}$ and analyzed by flow cytometry for the expression of HLA-ABC (a),
HLA-DR (b), CD86 (c), CD70 (d), ICAM-1 (e), and Fas (f). Heat maps display the mean of the geometric mean fluorescent intensity (MFI) of each marker divided by the MFI of matched isotype control antibodies (relative expression). $n=2$.

\section{Transgene expressing LOAd viruses enhance T cell activation in multiple myeloma co-cultures}

The immunological assessment of the armed LOAd viruses for $\mathrm{MM}$ treatment would ideally require an immunocompetent syngeneic mouse model. However, as the receptor for viral entry, CD46, is not expressed in murine cells and adenoviruses cannot replicate in murine cells [31], it is impossible to evaluate this therapy fully in these models, even if a virus construct with murine transgenes would be used. Hence, we examined in an in vitro model if LOAd viruses can activate immune cells conditioned in the presence of MM cells. For this, a co-culture of MM cells together with healthy donor PBMCs was set-up and $24 \mathrm{~h}$ later infected with LOAd viruses. We tested this experimental set-up with two of the six MM cell lines, L363 and U266-84, in order to examine if there is any difference in the magnitude of immune cell activation dependent on the virus replication and subsequent phenotype changes in these cell lines. L363 cells allowed efficient virus replication (average across all cell lines), whereas the replication was lowest in U266-84 cells. In addition, L363 cells expressed the $\mathrm{T}$ cell costimulatory molecule CD86 and upregulated HLA-DR expression upon infection with the armed viruses. In contrast, U266-84 cells did not express CD86 and displayed the greatest reductions in CD70 and ICAM-1 upon LOAd infection. The co-cultures were analyzed with flow cytometry analysis $48 \mathrm{~h}$ post infection. As the majority of cells in culture were $\mathrm{CD} 3+$, the $\mathrm{T}$ cell compartment was investigated further. The gating strategy for flow cytometry analysis is shown in Supplemental Fig. S9. In general, coculture with either MM cell line altered the CD8+/CD4+ ratio towards CD8 $+\mathrm{T}$ cells (Fig. 7a). However, presence of MM cells also expanded CD25+ CD127- regulatory $\mathrm{T}$ cells (Tregs), but these cells were reduced in LOAdinfected cultures (Fig. 7b). CD8+ and CD4+ T cells were further analyzed for phenotype changes based on CD45RA and CCR7 expression. Interestingly, CD45RA-CCR7+ central memory $\mathrm{T}$ cells were increased in both subsets in particular in cultures with LOAd703-infected cells (Fig. $7 \mathrm{~d})$. The activation status of $\mathrm{T}$ cells was assessed by checking the expression of activation markers CD69, PD-1, 


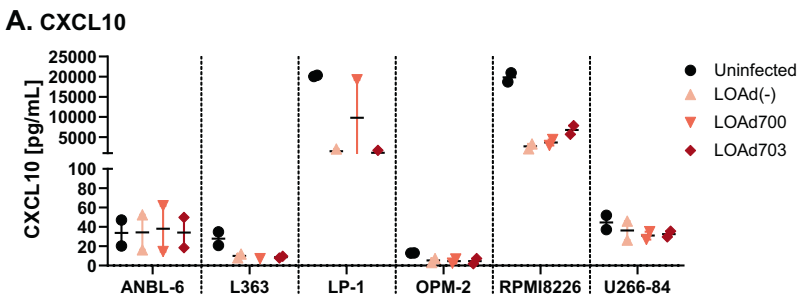

B. IL-8

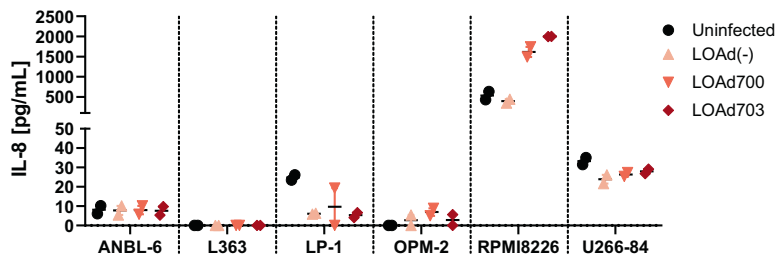

C. CCL2

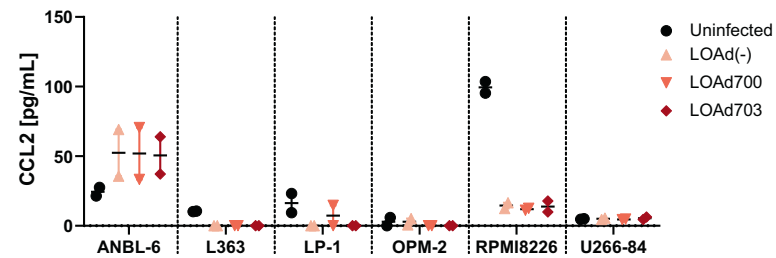

D. IL-2R $\alpha$

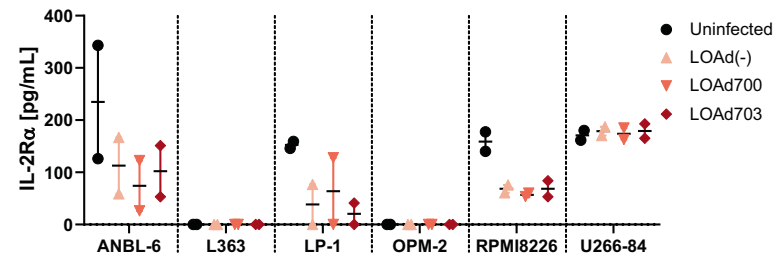

E. CCL3

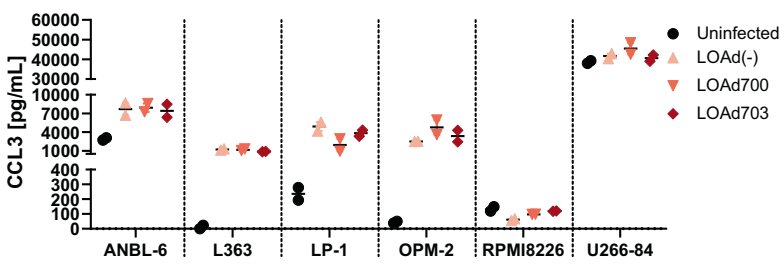

Fig. 6 Soluble immune markers in supernatants of LOAd-infected multiple myeloma cells. Cells were infected with $100 \mathrm{MOI}$ of LOAd (-), LOAd700 and LOAd703 or left uninfected. Forty-eight hours post infection, cell culture supernatants were harvested and analyzed by MSD multiplex assay for the presence of CXCL10 (a), IL-8 (b), CCL2 (c), sIL-2R $\alpha(\mathbf{d})$, and CCL3 (e). Graphs show the mean concentrations $\pm \mathrm{SEM}$ of the respective marker in $\mathrm{pg} / \mathrm{mL}$. $n=2$.

and degranulation marker CD107a (Fig. 7e-g). These markers were overall upregulated in the presence of LOAd virus infection with the highest level noted in the LOAd703 group. Likewise, cells activated by LOAd703 released IFN$\gamma$ to a significantly greater extent than cells infected with LOAd700 or LOAd(-), or uninfected controls (Fig. 7c). Overall, the $\mathrm{T}$ cell activation profile was comparable between the two cell lines used. Nevertheless, CD69 and IFN $\gamma$ expression levels were slightly lower in co-cultures with U266-84 cells, which is in agreement with the lower levels of co-stimulatory molecules and reduced viral replication in this cell line.

\section{Discussion}

Even though MM remains a largely incurable disease, treatment regimens including immunomodulatory drugs, such as lenalidomide, have clearly improved the outcome of patients [32]. Lenalidomide not only causes direct cytotoxicity in MM, but also has pleiotropic effects on the immune compartment [33]. Therapies targeting the immune system may be of particular interested in MM as patients with MM display notably impaired immune cell functions as seen by their increased susceptibility to infections [34]. Thus, this study aimed to determine if immunostimulatory oncolytic virotherapy within the LOAd platform may be a suitable treatment option for MM. Given that LOAd viruses are serotype Ad5/35 chimera, their serotype 35 fiber makes it possible to infect cells of hematopoietic origin via CD46. In fact, malignant plasma cells have been found to express CD46 to a much higher degree than normal hematopoietic cells [9]. This increased CD46 expression in MM cells has been shown to be regulated by p53 deficiency [35]. Serotype 35 adenoviruses have been reported to bind to MM cells up to 100 times better than other serotypes, whereas serotype 5 adenoviruses have showed more potent replication and MM cell killing [36]. Therefore, the chimeric serotype of LOAd viruses should facilitate both features in MM.

Indeed, all MM cell lines tested were very sensitive to viral infection at even low virus to cell ratio and efficiently killed. Also the replication-deficient Ad5/35 Mock virus could seemingly alter the cell metabolism of infected cells, which is the readout of the MTS viability assay used in the killing studies. Nevertheless, LOAd-infected MM cells were killed via oncolysis as seen by an up to 1000 fold increase of adenoviral DNA post infection. The minor replication of Mock virus observed in the MM cell lines may have been due to the potential presence of low numbers of wild type virus (RCA) in the Mock virus batch [25]. LOAd viruses were also able to control tumor growth through oncolysis in an in vivo xenograft model when injected intratumorally. There was no difference in the in vivo oncolytic function between $\operatorname{LOAd}(-)$ and LOAd703 expressing CD40L and 4-1BBL. Fernandes et al. observed enhanced tumor reductions in a RPMI-8226 xenograft model with a CD40L expressing oncolytic adenovirus [20]. However, the RPMI-8226 cells used herein had a reduced CD40 expression (6\%) compared to their study (50\%). Therefore, the lack of CD40/CD40L signaling inducing apoptosis [37] in the cells may be the reason for these contrasting results. Intravenous injections failed to 


\section{A. $\mathrm{CD} 8+/ \mathrm{CD} 4+$ ratio}

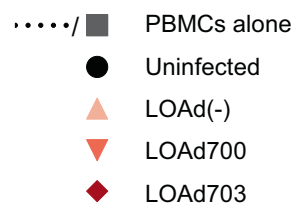

D. Central memory T cells
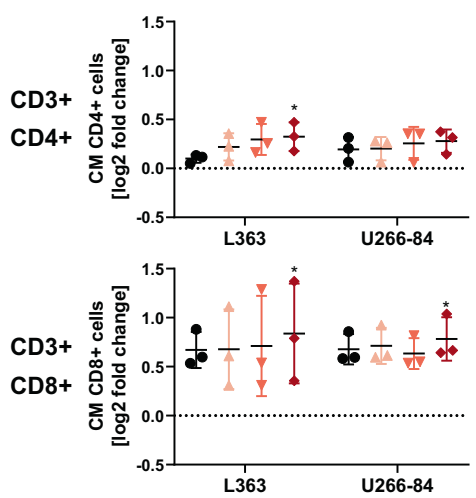

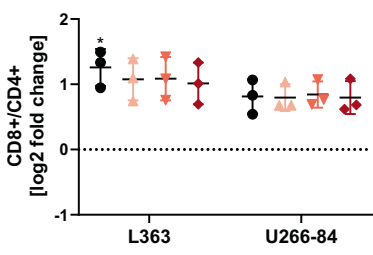

E. CD69+
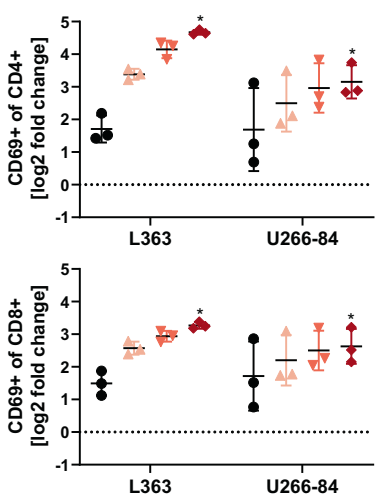
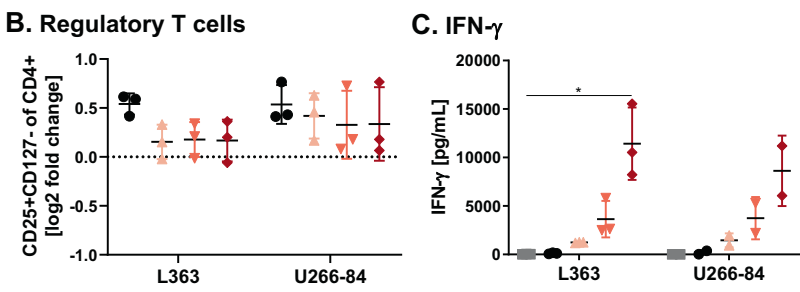

F. PD-1+

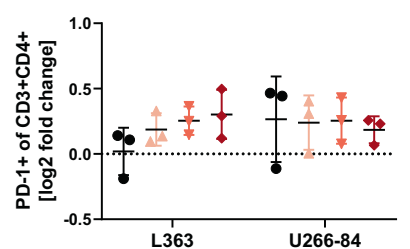

G. CD107a+
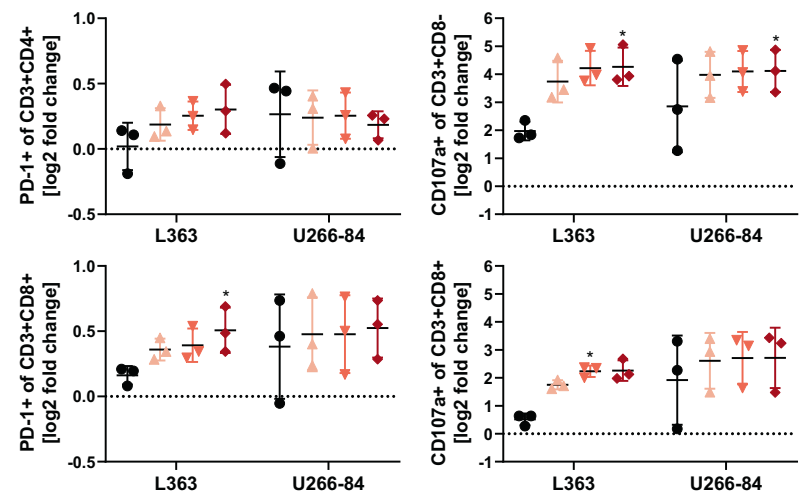

Fig. 7 LOAd viruses activate T cells in a co-culture of PBMC and multiple myeloma cells. Peripheral blood mononuclear cells (PBMCs) derived from healthy donor buffy coats $(n=3)$ were cocultured with L363 or U-266-84 cells ( $2: 1$ ratio; $1 \times 10^{6}$ cells $\left./ \mathrm{mL}\right)$ and $24 \mathrm{~h}$ later infected with 100 MOI of LOAd(-), LOAd700 and LOAd703 or left uninfected. Forty-eight hours post infection, cells were analyzed by flow cytometry for $\mathrm{T}$ cell markers and culture

control tumor growth, which is likely due to reduced delivery of virus particles to the actual tumor site upon intravenous injection. In humans, intravenous injections may be problematic due to the fact that adenoviruses are non-enveloped viruses, which can be readily cleared from the blood through opsonization by antibodies and complement [38] before reaching the tumor site. Thus, intratumoral delivery of LOAd viruses is favorable. Regardless, LOAd703 seemed to have a slightly better effect initially in the intravenous setting. Human 4-1BBL expressed by LOAd703 can slightly cross react with murine 4-1BB, whereas human $\mathrm{CD} 40 \mathrm{~L}$ cannot cross react with murine CD40 [26]. Therefore, the initial reduction in tumor growth by LOAd703 compared with $\operatorname{LOAd}(-)$ may be explained by 4-1BBL-mediated activation of innate immune cells, which are present in the nude mice used. Unfortunately, there is currently no suitable model available to analyze both the oncolytic and immunostimulatory function of LOAd viruses in vivo. Therefore, all investigations in regard to the effect of the transgenes were evaluated in vitro.

Next, we explored the transgenes effects on the MM cells as well as co-cultured immune cells. CD40L expression was observed upon infection with LOAd700 and LOAd703 and 4-1BBL expression was highly induced in all LOAd703infected cells. Since malignant plasma cells express similar supernatants were analyzed for IFN- $\gamma$ by ELISA. Flow cytometry results $(\mathbf{a}, \mathbf{b}, \mathbf{d}-\mathbf{g})$ are displayed as mean $\log 2$ fold change of percentage positive cells to PBMCs cultured alone (dotted line) \pm SD . c Mean IFN- $\gamma$ concentrations $(\mathrm{pg} / \mathrm{mL}) \pm \mathrm{SD}$. Statistically differences were analyzed with Friedman test followed by Dunn's multiple comparisons test $(* p<0.05)$.

molecules as professional antigen-presenting cells [16], we hypothesized that infection with CD40L expressing LOAd viruses could further alter the phenotype of MM cells as seen by others with CD40 stimulating therapy [19, 20, 39]. For example, in a study by Bashey et al., CD40 expressing MM cells infected with a CD40L encoding adenovirus upregulated the expression of HLA-DR, ICAM-1, and CD70 and could stimulate $\mathrm{T}$ cell proliferation in allogeneic mixed lymphocyte reactions [39]. The MM cell lines used in this study were found to be CD40 negative, apart from a weak CD40 expression in RPMI-8226 cells, and infection with LOAd700 or LOAd703 did not upregulate the abovementioned markers. In fact, MHC molecules as well as CD70 and ICAM-1 were rather downregulated upon LOAd infection in general, indicating that transcription levels may in some way be decreased due to the viral replication process. Downregulation of ICAM-1 could also be beneficial as adhesion molecules are known to mediate the interaction between $\mathrm{MM}$ and stromal cells, thereby facilitating MM growth and survival [40]. Also, high ICAM-1 expression has been connected to advanced disease and resistance to chemotherapy [41, 42]. Similarly, high CD70 expression has been detected on MM cells and been proposed as a therapeutic target [43]. In concert with these downregulations, an increase in the expression of the apoptosis receptor Fas was observed, which was most 
apparent in LOAd700 and LOAd703 infected cells. Hence, this may facilitate Fas/FasL mediated killing of the MM cells by $\mathrm{T}$ cells. In agreement, Fernandes et al. also observed an upregulation of Fas in MM cells infected with an oncolytic adenovirus encoding for CD40L [20].

Most investigated soluble immune markers released by MM cells were either stable or primarily reduced upon LOAd infection. These included CXCL10, IL-8, CCL2, and IL-2R $\alpha$. CXCL10 is generally seen as a pro-inflammatory chemokine, which is essential for the recruitment of Th1 lymphocytes to the tumor site [44]. However, its receptor CXCR3 is also overexpressed on MM cells and signaling through the receptor has been shown to promote chemotaxis and secretion of matrix metalloproteinases, which drive tumor invasion [45]. CCL2 can be induced by the MM growth factor IL-6 [46] and high CCL2 plasma levels in MM patients have been associated with angiogenesis and advanced disease [47]. Likewise, MM patients display increased levels of soluble IL-2R $\alpha$, which have been found to correlate negatively with progression-free survival [30]. CCL3 is another chemokine, which has been associated with poor prognosis and bone disease in MM patients [48]. But in contrast to the other factors, CCL3 was actually increased in the majority of cell lines post LOAd infection. Nevertheless, CCL3 could potentially also have a positive role in facilitating antitumor immune responses by recruiting DCs to the tumor site $[49,50]$, especially in combination with the pro-inflammatory environment commonly established with oncolytic viruses [7]. Of note, we have previously shown that both LOAd700 and LOAd703 are able to potently activate immature DCs $[11,15]$, which may be a crucial feature for establishing immune responses against MM cells as impaired DC functions have been frequently described in MM patients [51].

The immune dysregulation observed in MM patients also encompasses alterations in the $\mathrm{T}$ cell compartment [34], including shifts in the Th1/Th2 balance [52] and increased Treg levels [53]. Hence, we investigated the effect of LOAd viruses on different $\mathrm{T}$ cell subsets and their activation status in $\mathrm{MM}$ co-cultures. The $\mathrm{CD} 8+/ \mathrm{CD} 4+\mathrm{T}$ cell ratio was increased upon co-culture with either MM cell line and was left unchanged upon addition of LOAd viruses. As expected, Tregs were likewise increased in tumor co-cultures, but the levels were reduced again when cells were infected with LOAd viruses. When analyzing the $\mathrm{T}$ cell phenotype, a significant increase in central memory $\mathrm{T}$ cells was noted in samples infected with LOAd703. Higher levels of memory $\mathrm{T}$ cells is generally associated with a favorable outcome across cancer types [54] and lenalidomide has also been suggested to partly exert its effect through polarizing $\mathrm{T}$ cells into memory $\mathrm{T}$ cells [55]. Zelle-Rieser et al. analyzed the exhaustion profile and functionality of $\mathrm{T}$ cells from MM patients and they demonstrated that $\mathrm{T}$ cells at the tumor site were functionally impaired as they failed to both increase the degranulation marker CD107a and to produce IFN $\gamma$ in response to stimulation [56]. Upon infection with LOAd viruses, both $\mathrm{CD} 4+$ and $\mathrm{CD} 8+\mathrm{T}$ cells were able to highly upregulate CD107a as well as the activation marker CD69 in co-culture with MM cells. The highest upregulation was typically observed with LOAd703, which is encoding both CD40L and 4-1BBL. In addition, LOAd703 induced significantly higher IFN $\gamma$ levels than all other conditions, which is likely due to the additional stimulation of $\mathrm{T}$ cells through 4-1BBL/4-1BB signaling [57].

Overall, oncolytic virotherapy within the LOAd platform was shown to be a suitable candidate for the treatment of MM. Typical features of MM cells, such as overexpression of CD46 [9] and a dysregulated retinoblastoma pathway [12-14], enabled LOAd viruses to infect and kill a variety of MM cell lines, both in vitro and in vivo. Treatment in vivo was most effective when injected intratumorally. Even though MM is a systemic disease and intravenous injections may be desired, the patient group that has the biggest unmet clinical need also presents with extramedullary tumor sites, which may serve as a target lesion for LOAd injection. Interestingly, LOAd infection in MM cells resulted in the downregulation of markers associated with $\mathrm{MM}$ growth and survival and increased their immunogenicity by upregulating Fas. Most strikingly, LOAd viruses, in particular LOAd703, were able to activate and polarize $\mathrm{T}$ cells towards a Th1 response in a co-culture with MM cells. These results warrant further investigations of LOAd703 in $\mathrm{MM}$ and it would be of interest to explore the effect of the transgenes on the tumor stroma.

Acknowledgements This work was financially supported by grants from The Swedish Cancer Society, The Swedish Research Council and a contract research grant from Lokon Pharma AB.

Author contributions Conceptualization: J.W., E.E., and A.L.; Methodology: J.W., E.E., and A.L.; Formal Analysis: J.W. and S.N.; Investigation: J.W., S.N. and A.-C.H.; Resources: H.J.W. and A.L.; Writing - Original draft: J.W. and S.N.; Writing-Review and editing: J.W., S.N., A.-C.H., H.J.W, E.E. and A.L.; Visualization: J.W. and S. N.; Supervision: A.L.; Project administration: J.W., E.E., and A.L.; Funding acquisition: A.L.

\section{Compliance with ethical standards}

Conflict of interest Dr. Loskog discloses conflicts of interest connected to the data presented in this paper. Dr. Loskog is the CEO and board member of Lokon Pharma $\mathrm{AB}$ and holds a royalty agreement and a contract research agreement with Lokon Pharma $\mathrm{AB}$ at her adjunct position at Uppsala University. The other authors have no conflicts of interest to disclose.

Publisher's note Springer Nature remains neutral with regard to jurisdictional claims in published maps and institutional affiliations. 
Open Access This article is licensed under a Creative Commons Attribution 4.0 International License, which permits use, sharing, adaptation, distribution and reproduction in any medium or format, as long as you give appropriate credit to the original author(s) and the source, provide a link to the Creative Commons license, and indicate if changes were made. The images or other third party material in this article are included in the article's Creative Commons license, unless indicated otherwise in a credit line to the material. If material is not included in the article's Creative Commons license and your intended use is not permitted by statutory regulation or exceeds the permitted use, you will need to obtain permission directly from the copyright holder. To view a copy of this license, visit http://creativecommons. org/licenses/by/4.0/.

\section{References}

1. Bergin K, McQuilten Z, Moore E, Wood E, Spencer A. Myeloma in the real world: What is really happening? Clin Lymphoma Myeloma Leuk 2017;17:133-44.

2. Kazandjian D. Multiple myeloma epidemiology and survival: a unique malignancy. Semin Oncol 2016;43:676-81.

3. Pour L, Sevcikova S, Greslikova H, Kupska R, Majkova P, Zahradova L, et al. Soft-tissue extramedullary multiple myeloma prognosis is significantly worse in comparison to bone-related extramedullary relapse. Haematologica 2014;99:360-4.

4. Usmani SZ, Heuck C, Mitchell A, Szymonifka J, Nair B, Hoering A, et al. Extramedullary disease portends poor prognosis in multiple myeloma and is over-represented in high-risk disease even in the era of novel agents. Haematologica 2012;97:1761-7.

5. Rafei H, Haroun F, Tabbara IA. Novel immunotherapeutic agents for the treatment of multiple myeloma. Am J Clin Oncol 2019;42:317-29.

6. Rodriguez-Otero P, Paiva B, Engelhardt M, Prosper F, San Miguel JF. Is immunotherapy here to stay in multiple myeloma? Haematologica 2017;102:423-32.

7. Kaufman HL, Kohlhapp FJ, Zloza A. Oncolytic viruses: a new class of immunotherapy drugs. Nat Rev Drug Discov 2015;14:642-62.

8. Calton C, Kelly K, Anwer F, Carew J, Nawrocki S. Oncolytic viruses for multiple myeloma therapy. Cancers 2018;10:198.

9. Ong HT, Timm MM, Greipp PR, Witzig TE, Dispenzieri A, Russell SJ, et al. Oncolytic measles virus targets high CD46 expression on multiple myeloma cells. Exp Hematol 2006;34:713-20.

10. Yotnda P, Onishi H, Heslop HE, Shayakhmetov D, Lieber A, Brenner M, et al. Efficient infection of primitive hematopoietic stem cells by modified adenovirus. Gene Therapy. 2001;8:930-7.

11. Eriksson E, Moreno R, Milenova I, Liljenfeldt L, Dieterich LC, Christiansson L, et al. Activation of myeloid and endothelial cells by CD40L gene therapy supports T-cell expansion and migration into the tumor microenvironment. Gene Ther 2017;24:92-103.

12. Dao DD, Sawyer JR, Epstein J, Hoover RG, Barlogie B, Tricot G. Deletion of the retinoblastoma gene in multiple myeloma. Leukemia 1994;8:1280-4.

13. Juge-Morineau N, Mellerin MP, Francois S, Rapp MJ, Harousseau JL, Amiot M, et al. High incidence of deletions but infrequent inactivation of the retinoblastoma gene in human myeloma cells. Br J Haematol 1995;91:664-7.

14. Zandecki M, Facon T, Preudhomme C, Vanrumbeke M, Vachee A, Quesnel B, et al. The retinoblastoma gene (RB-1) status in multiple myeloma: a report on 35 cases. Leuk Lymphoma 1995;18:497-503.

15. Eriksson E, Milenova I, Wenthe J, Stahle M, Leja-Jarblad J, Ullenhag G, et al. Shaping the tumor stroma and sparking immune activation by CD40 and 4-1BB signaling induced by an armed oncolytic virus. Clin Cancer Res 2017;23:5846-57.
16. Yi Q, Dabadghao S, Osterborg A, Bergenbrant S, Holm G. Myeloma bone marrow plasma cells: evidence for their capacity as antigen-presenting cells. Blood 1997;90:1960-7.

17. Kato K, Cantwell MJ, Sharma S, Kipps TJ. Gene transfer of CD40-ligand induces autologous immune recognition of chronic lymphocytic leukemia B cells. J Clin Invest 1998;101:1133-41.

18. Wierda WG, Cantwell MJ, Woods SJ, Rassenti LZ, Prussak CE, Kipps TJ. CD40-ligand (CD154) gene therapy for chronic lymphocytic leukemia. Blood 2000;96:2917-24.

19. Dotti G. Adenovector-induced expression of human-CD40-ligand (hCD40L) by multiple myeloma cells: a model for immunotheraphy. Exp. Hematol. 2001;29:952-61.

20. Fernandes MS, Gomes EM, Butcher LD, Hernandez-Alcoceba R, Chang D, Kansopon J, et al. Growth inhibition of human multiple myeloma cells by an oncolytic adenovirus carrying the CD40. Ligand Transgene. 2009;15:4847-56.

21. Jernberg-Wiklund H, Nilsson K. Multiple myeloma cell lines. In: Masters JRW, Palsson BO, editors. Human cell culture: cancer cell lines part 3: leukemias and lymphomas. Dordrecht: Springer; 2002. p. 81-155.

22. Danielsson A, Dzojic H, Nilsson B, Essand M. Increased therapeutic efficacy of the prostate-specific oncolytic adenovirus Ad[I/ PPT-E1A] by reduction of the insulator size and introduction of the full-length E3 region. Cancer Gene Ther. 2008;15:203-13.

23. Danielsson A, Elgue G, Nilsson BM, Nilsson B, Lambris JD, Totterman TH, et al. An ex vivo loop system models the toxicity and efficacy of PEGylated and unmodified adenovirus serotype 5 in whole human blood. Gene Ther 2010;17:752-62.

24. Fishelson Z, Donin N, Zell S, Schultz S, Kirschfink M. Obstacles to cancer immunotherapy: expression of membrane complement regulatory proteins (mCRPs) in tumors. Mol Immunol 2003;40:109-23.

25. Lochmuller H, Jani A, Huard J, Prescott S, Simoneau M, Massie $\mathrm{B}$, et al. Emergence of early region 1-containing replicationcompetent adenovirus in stocks of replication-defective adenovirus recombinants (delta E1 + delta E3) during multiple passages in 293 cells. Hum Gene Ther. 1994;5:1485-91.

26. Bossen C, Ingold K, Tardivel A, Bodmer JL, Gaide O, Hertig S, et al. Interactions of tumor necrosis factor (TNF) and TNF receptor family members in the mouse and human. J Biol Chem 2006;281:13964-71.

27. Aggarwal R, Ghobrial IM, Roodman GD. Chemokines in multiple myeloma. Exp Hematol 2006;34:1289-95.

28. Cao Y, Luetkens T, Kobold S, Hildebrandt Y, Gordic M, Lajmi N, et al. The cytokine/chemokine pattern in the bone marrow environment of multiple myeloma patients. Exp Hematol 2010;38:860-7.

29. Freire-de-Lima L, Nardy A, Ramos-Junior ES, Conde L, Santos Lemos J, da Fonseca LM, et al. Multiple myeloma cells express key immunoregulatory cytokines and modulate the monocyte migratory response. Front Med. 2017;4:92.

30. Wang L, Wang JH, Liu WJ, Wang WD, Wang H, Chen XQ, et al. High level of soluble interleukin-2 receptor in serum predicts treatment resistance and poor progression-free survival in multiple myeloma. Ann Hematol 2017;96:2079-88.

31. Jogler C, Hoffmann D, Theegarten D, Grunwald T, Uberla K, Wildner O. Replication properties of human adenovirus in vivo and in cultures of primary cells from different animal species. J Virol. 2006;80:3549-58.

32. Holstein SA, McCarthy PL. Immunomodulatory drugs in multiple myeloma: mechanisms of action and clinical experience. Drugs. 2017;77:505-20.

33. Kotla V, Goel S, Nischal S, Heuck C, Vivek K, Das B, et al. Mechanism of action of lenalidomide in hematological malignancies. J Hematol Oncol. 2009;2:36.

34. Pratt G, Goodyear O, Moss P. Immunodeficiency and immunotherapy in multiple myeloma. Br J Haematol 2007;138:563-79. 
35. Lok A, Descamps G, Tessoulin B, Chiron D, Eveillard M, Godon $\mathrm{C}$, et al. p53 regulates CD46 expression and measles virus infection in myeloma cells. Blood Adv. 2018;2:3492-505.

36. Senac JS, Doronin K, Russell SJ, Jelinek DF, Greipp PR, Barry MA. Infection and killing of multiple myeloma by adenoviruses. Hum Gene Ther. 2010;21:179-90.

37. Eliopoulos AG, Davies C, Knox PG, Gallagher NJ, Afford SC, Adams DH, et al. CD40 induces apoptosis in carcinoma cells through activation of cytotoxic ligands of the tumor necrosis factor superfamily. Mol Cell Biol. 2000;20:5503-15.

38. Xu Z, Tian J, Smith JS, Byrnes AP. Clearance of adenovirus by Kupffer cells is mediated by scavenger receptors, natural antibodies, and complement. J Virol. 2008;82:11705-13.

39. Bashey A, Cantwell MJ, Kipps TJ. Adenovirus transduction to effect CD40 signalling improves the immune stimulatory activity of myeloma cells. Br J Haematol 2002;118:506-13.

40. Hideshima T, Mitsiades C, Tonon G, Richardson PG, Anderson $\mathrm{KC}$. Understanding multiple myeloma pathogenesis in the bone marrow to identify new therapeutic targets. Nat Rev Cancer. 2007;7:585-98.

41. Sampaio MS, Vettore AL, Yamamoto M, Chauffaille Mde L, Zago MA, Colleoni GW. Expression of eight genes of nuclear factor-kappa B pathway in multiple myeloma using bone marrow aspirates obtained at diagnosis. Histol Histopathol 2009;24:991-7.

42. Schmidmaier R, Morsdorf K, Baumann P, Emmerich B, Meinhardt G. Evidence for cell adhesion-mediated drug resistance of multiple myeloma cells in vivo. Int $\mathrm{J}$ Biol Markers. 2006;21:218-22.

43. McEarchern JA, Smith LM, McDonagh CF, Klussman K, Gordon KA, Morris-Tilden CA, et al. Preclinical characterization of SGN70, a humanized antibody directed against CD70. Clin Cancer Res. 2008;14:7763-72.

44. Liu M, Guo S, Stiles JK. The emerging role of CXCL10 in cancer. Oncol Lett. 2011;2:583-9.

45. Pellegrino A, Antonaci F, Russo F, Merchionne F, Ribatti D, Vacca A, et al. CXCR3-binding chemokines in multiple myeloma. Cancer Lett 2004;207:221-7.

46. Arendt BK, Velazquez-Dones A, Tschumper RC, Howell KG, Ansell SM, Witzig TE, et al. Interleukin 6 induces monocyte chemoattractant protein-1 expression in myeloma cells. Leukemia 2002;16:2142-7.
47. Valkovic T, Babarovic E, Lucin K, Stifter S, Aralica M, SeiliBekafigo I, et al. Plasma levels of monocyte chemotactic protein-1 are associated with clinical features and angiogenesis in patients with multiple myeloma. Biomed Res Int 2016;2016:7870590.

48. Terpos E, Politou M, Szydlo R, Goldman JM, Apperley JF, Rahemtulla A. Serum levels of macrophage inflammatory protein1 alpha (MIP-1alpha) correlate with the extent of bone disease and survival in patients with multiple myeloma. $\mathrm{Br} \mathrm{J}$ Haematol 2003;123:106-9.

49. Allen F, Bobanga ID, Rauhe P, Barkauskas D, Teich N, Tong C, et al. CCL3 augments tumor rejection and enhances CD8(+) T cell infiltration through $\mathrm{NK}$ and $\mathrm{CD} 103(+)$ dendritic cell recruitment via IFNgamma. Oncoimmunology 2018;7:e1393598.

50. Crittenden M, Gough M, Harrington K, Olivier K, Thompson J, Vile RG. Expression of inflammatory chemokines combined with local tumor destruction enhances tumor regression and long-term immunity. Cancer Res 2003;63:5505-12.

51. Brimnes MK, Svane IM, Johnsen HE. Impaired functionality and phenotypic profile of dendritic cells from patients with multiple myeloma. Clin Exp Immunol 2006;144:76-84.

52. Sharma A, Khan R, Joshi S, Kumar L, Sharma M. Dysregulation in $\mathrm{T}$ helper $1 / \mathrm{T}$ helper 2 cytokine ratios in patients with multiple myeloma. Leuk Lymphoma 2010;51:920-7.

53. Lad D, Huang Q, Hoeppli R, Garcia R, Xu L, Levings M, et al. Evaluating the role of Tregs in the progression of multiple myeloma. Leuk Lymphoma 2019;60:2134-42.

54. Jia Q, Yang Y, Wan Y. Tumor-infiltrating memory Tlymphocytes for prognostic prediction in cancer patients: a meta-analysis. Int J Clin Exp Med 2015;8:1803-13.

55. Busch A, Zeh D, Janzen V, Mugge LO, Wolf D, Fingerhut L, et al. Treatment with lenalidomide induces immunoactivating and counter-regulatory immunosuppressive changes in myeloma patients. Clin Exp Immunol 2014;177:439-53.

56. Zelle-Rieser $\mathrm{C}$, Thangavadivel $\mathrm{S}$, Biedermann $\mathrm{R}$, Brunner A, Stoitzner $\mathrm{P}$, Willenbacher E, et al. T cells in multiple myeloma display features of exhaustion and senescence at the tumor site. J Hematol Oncol 2016;9:116.

57. Wen T, Bukczynski J, Watts TH. 4-1BB ligand-mediated costimulation of human $\mathrm{T}$ cells induces CD4 and CD8 T cell expansion, cytokine production, and the development of cytolytic effector function. J Immunol. 2002;168:4897-906. 